\title{
The index of symmetry of a flag manifold
}

\author{
Fabio Podestà
}

\begin{abstract}
We study the index of symmetry of a compact generalized flag manifold $M=G / H$ endowed with an invariant Kähler structure. When the group $G$ is simple we show that the leaves of symmetry are irreducible Hermitian symmetric spaces which depend only on the invariant complex structure and we estimate their dimension.
\end{abstract}

\section{Introduction}

Recently Olmos, Reggiani and Tamaru ([8]) introduced the notion of index of symmetry for a Riemannian manifold. In particular given a Riemannian manifold $(M, g)$ on which the full group of isometries $G$ acts transitively, one can define the distribution of symmetry $\mathcal{D}$ which at each point $x \in M$ is given by the values at $x$ of all Killing vector fields $X$ with $\left.\nabla X\right|_{x}=0$, where $\nabla$ denotes the Levi Civita connection of $g$. The main motivation for introducing $\mathcal{D}$ is the fact that the index of symmetry $\imath_{s}(M):=\operatorname{dim} \mathcal{D}$ coincides with $\operatorname{dim} M$ precisely when $(M, g)$ is a symmetric space. Therefore the coindex $\operatorname{dim} M-\imath_{s}(M)$ can be viewed as a sort of "distance" of the manifold from being a Riemannian symmetric space. It is proved that the distribution $\mathcal{D}$ is actually integrable and that the maximal integral submanifolds, which we will call the leaves of symmetry, are Riemannian symmetric s paces which are embedded into $M$ as totally geodesic submanifolds.

In this work we will focus on compact generalized flag manifolds, namely compact homogeneous spaces $G / H$ where $G$ is a connected compact semisimple Lie group and $H$ is the centralizer in $G$ of a torus. It is well known that these manifolds exhaust all compact Kähler manifolds which admit a transitive semisimple compact Lie group of biholomorphic isometries. If $M=G / H$ is a such a manifold endowed with an invariant Kähler structure given by an invariant complex structure $J$ and a Kähler metric g compatible with $J$, then $(M, g)$ is de Rham irreducible if and only if $G$ is simple and in this case $G$ coincides (up to covering) with the full group of isometries of $(M, g)$ up to a few exceptions which can be listed (see §2). Our main result can be stated as follows.

Mathematics Subject Classification (2010): 53C30, 53C35.

Keywords: Homogeneous manifolds, flag manifolds, symmetric spaces. 
Theorem 1.1. Let $M=G / H$ be a compact generalized flag manifold endowed with a non-symmetric $G$-invariant Kähler structure $(g, J)$. Suppose that $G$ is simple and that it coincides with the full group of isometries of $g$. Then,

i) the leaves of symmetry are complex, totally geodesic submanifolds which are irreducible Hermitian symmetric spaces.

ii) There exists a compact Lie subgroup $H^{\prime} \supset H$ such that the fibers of the fibration $G / H \rightarrow G / H^{\prime}$ coincide with the leaves of symmetry. The subgroup $H^{\prime}$ depends only on the complex structure $J$ and not on the metric $g$.

iii) If $k$ denotes the co-index of symmetry (i.e., $\left.k=\operatorname{dim} G / H^{\prime}\right)$, then

$$
\operatorname{dim} G \leq \frac{1}{2} k(k-1) \quad \text { and } \quad k \geq 6
$$

with $k=6$ precisely when $\mathfrak{g}=\mathfrak{s u}(4)$ and $\mathfrak{h}=2 \mathbb{R} \oplus \mathfrak{s u}(2)$.

We first remark that when $G$ is not simple and splits (locally) as a product $G=G_{1} \times \cdots \times G_{r}$ of simple factors $G_{1}, \ldots, G_{r}$, then there are subgroups $H_{i} \subset G_{i}$ such that $M=G_{1} / H_{1} \times \cdots \times G_{r} / H_{r}$ biholomorphically and isometrically, so that the above theorem can be applied on each factor leading to the general description of the leaves of symmetry for any generalized flag manifold. The hypothesis that $G$ coincides (up to covering) with the full group of isometries is also not too restrictive, as the result due to Onishchick shows (see Theorem 2.1).

As stated in our main theorem, it is remarkable that the leaves of symmetry are irreducible symmetric spaces and that the subgroup $H^{\prime}$ depends exclusively on the invariant complex structure and not on the Kähler metric. In Remark 3.4 we give a simple and constructive way to identify the subgroup $H^{\prime}$ in terms of the highest root of the root system of the Lie algebra $\mathfrak{g}$ of $G$, equipped with the ordering corresponding to the invariant complex structure. Note also that in general the invariant complex structure on $M$ does not descend to an invariant (almost)-complex structure on $G / H^{\prime}$.

Note also that in [2] the authors prove an estimate for the co-index of symmetry from which we get $\operatorname{dim} G \leq \frac{1}{2} k(k+1)$, while the estimate (1.1) is finer in our setting. We remark that $k \geq 6$ improves the inequality $k \geq 2$ which is proved in a general setting in [2].

The paper is structured as follows. In Section 2 we give a brief survey on the basic structure of generalized flag manifolds and their invariant Kähler structures; we then explain the notion of index of symmetry and related geometric features. In Section 3 we give the proof of the main theorem 1.1.

Notation. For a compact Lie group, we denote its Lie algebra by the corresponding lowercase gothic letter. If a group $G$ acts on a manifold $M$, for every $X \in \mathfrak{g}$ we denote by $\hat{X}$ the corresponding vector field on $M$ induced by the $G$-action.

Acknowledgments. The author thanks D. V. Alekseevsky for valuable conversations. 


\section{Preliminaries}

In this section we will review some basic facts about generalized flag manifolds and the symmetry index.

\subsection{Generalized flag manifolds}

We consider a compact connected semisimple Lie group $G$ and a compact subgroup $H$ which coincides with the centralizer in $G$ of a torus. The homogeneous space $M=G / H$ is a generalized flag manifold and it can be equipped with invariant Kähler structures. We will now state some of the main properties of generalized flag manifolds, referring to [1] and [3] for a more detailed exposition.

We fix a maximal abelian subalgebra $\mathfrak{t} \subset \mathfrak{h}$ and the $B$-orthogonal decomposition $\mathfrak{g}=\mathfrak{h} \oplus \mathfrak{m}$. The subspace $\mathfrak{m}$ can be naturally identified with the tangent space $T_{o} M$, where $o:=[H] \in G / H$. If $R$ denotes the root system of $\mathfrak{g}^{\mathbb{C}}$ relative to the Cartan subalgebra $\mathfrak{t}^{\mathbb{C}}$, for every root $\alpha \in R$ the corresponding root space is given by $\mathfrak{g}_{\alpha}=\mathbb{C} \cdot E_{\alpha}$ and

$$
\mathfrak{h}^{\mathbb{C}}=\bigoplus_{\alpha \in R_{\mathfrak{h}}} \mathfrak{g}_{\alpha}, \quad \mathfrak{m}^{\mathbb{C}}=\bigoplus_{\alpha \in R_{\mathfrak{m}}} \mathfrak{g}_{\alpha},
$$

where $R_{\mathfrak{h}} \subset R$ is a closed subsystem of roots and $R_{\mathfrak{m}}:=R \backslash R_{\mathfrak{h}}$. The roots in $R_{\mathfrak{h}}$ are characterized by the fact that they vanish on the center $\mathfrak{c} \subseteq \mathfrak{t}$ of $\mathfrak{h}$. Observe that $\left(R_{\mathfrak{h}}+R_{\mathfrak{m}}\right) \cap R \subseteq R_{\mathfrak{m}}$.

Any $G$-invariant complex structure $J$ on $M$ induces an endomorphism $J \in$ $\operatorname{End}(\mathfrak{m})$ with $J^{2}=-$ Id. If we extend $J$ to $\mathfrak{m}^{\mathbb{C}}$ and we decompose $\mathfrak{m}^{\mathbb{C}}=\mathfrak{m}^{1,0} \oplus \mathfrak{m}^{0,1}$ into the sum of the $\pm i$ - eigenspaces of $J$, then the integrability of $J$ is equivalent to the fact that $\mathfrak{q}:=\mathfrak{h}^{\mathbb{C}} \oplus \mathfrak{m}^{1,0}$ is a subalgebra, actually a parabolic subalgebra of $\mathfrak{g}^{\mathbb{C}}$. Moreover it can be shown that $G$-invariant complex structures are in bijective correspondence with the invariant orderings of $R_{\mathfrak{m}}$, namely subsets $R_{\mathfrak{m}}^{+} \subset R_{\mathfrak{m}}$ such that

$$
R_{\mathfrak{m}}=R_{\mathfrak{m}}^{+} \cup\left(-R_{\mathfrak{m}}^{+}\right), \quad R_{\mathfrak{m}}^{+} \cap\left(-R_{\mathfrak{m}}^{+}\right)=\emptyset, \quad\left(R_{\mathfrak{h}}+R_{\mathfrak{m}}^{+}\right) \cap R \subset R_{\mathfrak{m}}^{+},
$$

the correspondence being given by $\mathfrak{m}^{1,0}=\bigoplus_{\alpha \in R_{\mathfrak{m}}^{+}} \mathfrak{g}_{\alpha}$. Invariant orderings are then in one-to-one correspondence with Weyl chambers in the center $\mathfrak{c}$ of $\mathfrak{h}$, namely connec-ted components of the set $\mathfrak{c} \backslash \bigcup_{\alpha \in R_{\mathfrak{m}}} \operatorname{ker}\left(\left.\alpha\right|_{\mathfrak{c}}\right)$, and an invariant ordering in $R_{\mathfrak{m}}$ can be combined with an ordering in $R_{\mathfrak{h}}$ to provide a standard ordering in $R$.

If we fix an invariant complex structure $J$ on $M$ (hence a Weyl chamber $C$ in $\mathfrak{c}$ ), we can endow $M$ with many $G$-invariant Kähler metrics which are Hermitian with respect to $J$. Actually, it can be proved that $G$-invariant symplectic structures, namely $G$-invariant non-degenerate closed two-forms, are in one-to-one correspondence with elements in the Weyl chambers in $\mathfrak{c}$. Indeed, if $\omega \in \Lambda^{2}(\mathfrak{m})$ is a symplectic form, then there exists $\xi$ in some Weyl chamber in $\mathfrak{c}$ such that

$$
\omega(X, Y)=B\left(\operatorname{ad}_{\xi} X, Y\right), \quad X, Y \in \mathfrak{m} .
$$

Moreover $\omega$ is the Kähler form of a Kähler metric $g$ with respect to the complex structure $J$ (i.e., $g:=\omega(\cdot, J \cdot)$ defines a Kähler metric) if and only if $\xi \in C$.

Finally, if $M=G / H$ is endowed with an invariant Kähler structure $(g, J)$ and $G={ }_{\text {loc }} G_{1} \times \cdots \times G_{k}$ is the decomposition into a product of simple factors, then $H$ 
splits accordingly as $H={ }_{\text {loc }} H_{1} \times \cdots \times H_{k}$ for $H_{i} \subset G_{i}$, and $M$ is biholomorphically isometric to the product of irreducible Kähler homogeneous spaces $M=M_{1} \times \cdots \times$ $M_{k}, M_{i}:=G_{i} / H_{i}$. The next result, due to Onishchik ([9]), deals with the basic question whether $\mathfrak{g}$ coincides with the full algebra of Killing vector fields.

Theorem 2.1. If $G$ is a compact connected simple Lie group and acts almost effectively on $M=G / H$, then $G$ coincides (up to a covering) with the identity component of the full isometry group $Q$, with the following exceptions:

(a) $M=\mathbb{C} P^{2 n+1}$ and $\mathfrak{g}=\mathfrak{s p}(n+1), \mathfrak{h}=\mathfrak{u}(1) \oplus \mathfrak{s p}(n), \mathfrak{q}=\mathfrak{s u}(2 n+2)$;

(b) $\mathfrak{g}=\mathfrak{s o}(2 n-1), \mathfrak{h}=\mathfrak{u}(n-1), \mathfrak{q}=\mathfrak{s o}(2 n), \quad n \geq 4$;

(c) $M=Q_{5}$ and $\mathfrak{g}=\mathfrak{g}_{2}, \mathfrak{h}=\mathfrak{u}(2), \mathfrak{q}=\mathfrak{s o}(7)$.

\subsection{The index of symmetry}

The concept of index of symmetry has been introduced in [8]. If $(M, g)$ is a Riemannian manifold and $\mathcal{K}(M, g)$ is the set of all Killing vector fields, at each point $x \in M$ we can define the subspace

$$
\mathfrak{p}^{x}:=\left\{X \in \mathcal{K}(M, g)|\nabla X|_{x}=0\right\},
$$

where $\nabla$ denotes the Levi Civita connection of $g$. The elements of $\mathfrak{p}^{x}$ are called transvections at $q$ and the symmetric isotropic subalgebra $\mathfrak{k}^{x}$ at $x$ is defined as the linear span of the commutators $[X, Y]$ with $X, Y \in \mathfrak{p}^{x}$. It is clear that

$$
\mathfrak{u}^{x}:=\mathfrak{k}^{x} \oplus \mathfrak{p}^{x}
$$

is an involutive Lie algebra. The symmetric subspace $\mathfrak{s}_{x} \subset T_{x} M$ is then defined as

$$
\mathfrak{s}_{x}:=\left\{\hat{X}_{x} \mid X \in \mathfrak{p}^{x}\right\}
$$

and the index of symmetry $\imath_{s}(M)$ is $\inf _{x \in M} \operatorname{dim} \mathfrak{s}_{x}$. When $M$ is homogeneous, the assignment $x \mapsto \mathfrak{s}_{x}$ defines a distribution which is proven to be integrable and autoparallel. The maximal integral leaves of this symmetry distribution are Riemannian symmetric spaces which are embedded in $M$ as totally geodesic submanifolds. One of the main reasons for considering the index of symmetry is the well-known fact that $\imath_{s}(M)=\operatorname{dim} M$ if and only if $M$ is a symmetric space (see [8]).

\section{The main result}

In this section we consider a generalized flag manifold $M=G / H$, where $G$ is a compact semisimple connected Lie group and we endow $M$ with a $G$-invariant Kähler structure, given by a complex structure $J$ and a Kähler metric $g$. We also keep the same notations as in the previous section.

We will also assume that the Lie algebra $\mathfrak{g}$ coincides with the algebra of the full isometry group and we fix a reductive decomposition

$$
\mathfrak{g}=\mathfrak{h} \oplus \mathfrak{m}, \quad[\mathfrak{h}, \mathfrak{m}] \subseteq \mathfrak{m} .
$$

We denote by $o:=[e H] \in G / H$ and by $\mathfrak{p}$ the subspace $\mathfrak{p}^{o} \subset \mathfrak{g}$. We first prove: 
Lemma 3.1. The subspace $\mathfrak{p} \subseteq \mathfrak{g}$ is $\operatorname{Ad}(H)$-invariant and contained in $\mathfrak{m}$. Therefore $\mathfrak{p} \cong \mathfrak{s}_{O}$ and it is complex, i.e., $J\left(\mathfrak{s}_{O}\right)=\mathfrak{s}_{O}$.

Proof. If $h \in H$ and $X \in \mathfrak{p}$, then $\widehat{\operatorname{Ad}(h) X}=h_{*} \hat{X}$ and therefore for every $w \in T_{o} M$ we have

$$
\left.\nabla_{w} \widehat{\operatorname{Ad}(h) X}\right|_{o}=\left.d h^{-1}\right|_{o}\left(\nabla_{d h^{-1} w} \hat{X}\right)=0,
$$

hence $\operatorname{Ad}(h) X \in \mathfrak{p}$. If we fix a maximal abelian subalgebra $\mathfrak{t} \subseteq \mathfrak{h}$ then $[\mathfrak{t}, \mathfrak{p}] \subseteq \mathfrak{p}$. This implies that $\mathfrak{p}^{\mathbb{C}}$ splits as the sum of root spaces and therefore $\mathfrak{p}^{\mathbb{C}}=\left(\mathfrak{p}^{\mathbb{C}} \cap \mathfrak{h}^{\mathbb{C}}\right) \oplus$ $\left(\mathfrak{p}^{\mathbb{C}} \cap \mathfrak{m}^{\mathbb{C}}\right)$. Since $\mathfrak{p} \cap \mathfrak{h}=\{0\}$ by well known properties of Killing vector fields, we see that $\mathfrak{p}^{\mathbb{C}} \subseteq \mathfrak{m}^{\mathbb{C}}$, hence $\mathfrak{p} \subseteq \mathfrak{m}$.

We now prove that $\mathfrak{p}$ is complex. Recall (see e.g. [1]) the fact that the $\mathfrak{h}$-module $\mathfrak{m}$ splits as the sum of mutually inequivalent submodules $\mathfrak{m}=\bigoplus_{i=1}^{k} \mathfrak{m}_{i}$ each of which is therefore $J$-stable. Since $\mathfrak{p}$ is an $\mathfrak{h}$-submodule, it is the sum of a certain number of submodules $\mathfrak{m}_{j}$ and therefore it is $J$-stable.

Keeping the same notations, we fix a Cartan subalgebra $\mathfrak{t}^{\mathbb{C}} \subset \mathfrak{g}^{\mathbb{C}}$ and an ordering of the corresponding root system $R$ so that

$$
\mathfrak{m}^{1,0}=\bigoplus_{\alpha \in R_{\mathfrak{m}}^{+}} \mathfrak{g}_{\alpha} .
$$

The submodule $\mathfrak{p}^{\mathbb{C}}$ is also the sum of certain root spaces, say $\mathfrak{p}^{\mathbb{C}}=\bigoplus_{\alpha \in R_{\mathfrak{p}}} \mathfrak{g}_{\alpha}$, where $R_{\mathfrak{p}} \subset R_{\mathfrak{m}}$ with $R_{\mathfrak{p}}=-R_{\mathfrak{p}}$. We now recall the well-known expression for the Levi Civita connection of an invariant metric on a reductive homogeneous space (see e.g. [7]). If $X, Y, U \in \mathfrak{m}$, then

$$
-2 g_{o}\left(\nabla_{\hat{Y}} \hat{X}, \hat{U}\right)=\left\langle[Y, X]_{\mathfrak{m}}, U\right\rangle+\left\langle[X, U]_{\mathfrak{m}}, Y\right\rangle+\left\langle[Y, U]_{\mathfrak{m}}, X\right\rangle
$$

where $\langle\cdot, \cdot\rangle$ is the $\operatorname{Ad}(H)$-invariant scalar product on $\mathfrak{m}$ corresponding to $g_{o}$. Therefore, $X \in \mathfrak{p}$ if and only if for every $Y, U \in \mathfrak{m}$ we have

$$
\left\langle[Y, X]_{\mathfrak{m}}, U\right\rangle+\left\langle[X, U]_{\mathfrak{m}}, Y\right\rangle+\left\langle[Y, U]_{\mathfrak{m}}, X\right\rangle=0 .
$$

We can extend (3.2) $\mathbb{C}$-linearly and we can also suppose that $X=E_{\alpha}$ for some $\alpha \in R_{\mathfrak{p}}^{+}$, where $R_{\mathfrak{p}}^{+}=R_{\mathfrak{p}} \cap R_{\mathfrak{m}}^{+}$. Here $\left\{E_{\alpha}\right\}_{\alpha \in R}$ denotes the standard Chevalley basis of the root spaces (see e.g. Theorem 5.5 in [5]). Equation (3.2) implies that $E_{\alpha} \in \mathfrak{p}^{\mathbb{C}}$ if and only if for every roots $\beta, \gamma \in R_{\mathfrak{m}}$ we have

$$
\left\langle\left[E_{\beta}, E_{\alpha}\right]_{\mathfrak{m}}, E_{\gamma}\right\rangle+\left\langle\left[E_{\alpha}, E_{\gamma}\right]_{\mathfrak{m}}, E_{\beta}\right\rangle+\left\langle\left[E_{\beta}, E_{\gamma}\right]_{\mathfrak{m}}, E_{\alpha}\right\rangle=0 .
$$

We recall now that for $\beta, \gamma \in R_{\mathfrak{m}}$ we have

$$
\begin{aligned}
\left\langle E_{\beta}, E_{\gamma}\right\rangle & =-i \epsilon_{\beta} \cdot\left\langle J E_{\beta}, E_{\gamma}\right\rangle=-i \epsilon_{\beta} \cdot \omega\left(E_{\beta}, E_{\gamma}\right) \\
& =-i \epsilon_{\beta} \cdot B\left(\left[\xi, E_{\beta}\right], E_{\gamma}\right)=-i \epsilon_{\beta} \cdot \beta(\xi) \cdot B\left(E_{\beta}, E_{\gamma}\right),
\end{aligned}
$$

where $\epsilon_{\beta}= \pm 1$ according to $\beta \in R_{\mathfrak{m}}^{ \pm}$. Therefore $\left\langle E_{\beta}, E_{\gamma}\right\rangle=0$ unless $\gamma=-\beta$ and

$$
\left\langle E_{\beta}, E_{-\beta}\right\rangle=-i \epsilon_{\beta} \cdot \beta(\xi) .
$$


It follows that equation (3.3) is significant only when $\beta+\gamma=-\alpha$, and in this case,

$$
N_{\beta, \gamma} \cdot\left\langle E_{-\alpha}, E_{\alpha}\right\rangle+N_{\alpha, \gamma} \cdot\left\langle E_{-\beta}, E_{\beta}\right\rangle+N_{\beta, \alpha} \cdot\left\langle E_{-\gamma}, E_{\gamma}\right\rangle=0 .
$$

Since $\alpha+\beta+\gamma=0$ we have that $N_{\alpha, \beta}=N_{\beta, \gamma}=N_{\gamma, \alpha}$ (see Lemma 5.1 in [5]), and therefore we must have

$$
\left\langle E_{-\alpha}, E_{\alpha}\right\rangle=\left\langle E_{-\beta}, E_{\beta}\right\rangle+\left\langle E_{-\gamma}, E_{\gamma}\right\rangle .
$$

Using (3.4), we see that (3.5) is equivalent to

$$
\left(\left(1+\epsilon_{\gamma}\right) \cdot \gamma+\left(1+\epsilon_{\beta}\right) \cdot \beta\right)(\xi)=0 .
$$

Now, $\alpha>0$, so that $\beta$ and $\gamma$ are both negative or have opposite sign. If $\beta, \gamma<0$ then (3.6) is automatic, while if, say, $\beta<0$ and $\gamma>0$, then (3.6) implies $\gamma(\xi)=0$. This contradicts the fact that $\gamma \in R_{\mathfrak{m}}$, while $\mathfrak{h}$ is the centralizer of $\xi$ in $\mathfrak{g}$. Therefore we conclude that $\alpha \in R_{\mathfrak{m}}^{+}$belongs to $R_{\mathfrak{p}}$ if and only if $\alpha \neq-\beta-\gamma$ with $\beta$ and $\gamma$ in $R_{\mathfrak{m}}$ with opposite signs or, equivalently, if and only if $\left(\alpha+R_{\mathfrak{m}}^{+}\right) \cap R=\emptyset$. This allows the following characterization:

Lemma 3.2. The subspace $\mathfrak{p}^{\mathbb{C}} \cap \mathfrak{m}^{1,0}$ coincides with the center $\mathfrak{z}$ of the nilpotent subalgebra $\mathfrak{m}^{1,0}$ of $\mathfrak{g}^{\mathbb{C}}$.

Proof. Indeed the center $\mathfrak{z}$ is spanned by root vectors $E_{\alpha}, \alpha \in R_{\mathfrak{m}}^{+}$, such that $\left[E_{\alpha}, E_{\beta}\right]=0$ for every $\beta \in R_{\mathfrak{m}}^{+}$and this is equivalent to saying that $\alpha+R_{\mathfrak{m}}^{+}$does not contain roots.

We remark that the subalgebra $\mathfrak{m}^{1,0}$ is the nilponent radical of the parabolic subalgebra $\overline{\mathfrak{q}}:=\mathfrak{h}^{\mathbb{C}} \oplus \mathfrak{m}^{1,0}$.

If we now put $\mathfrak{k}:=[\mathfrak{p}, \mathfrak{p}]$ and $\mathfrak{u}:=\mathfrak{p} \oplus \mathfrak{k}$, then $(\mathfrak{u}, \mathfrak{k})$ is a symmetric pair and we can prove the following lemma.

Lemma 3.3. If $\mathfrak{g}$ is simple, the pair $(\mathfrak{u}, \mathfrak{k})$ is an irreducible Hermitian symmetric pair. The Lie algebra $\mathfrak{u}$ is also simple.

Proof. The fact that the symmetric pair $(\mathfrak{u}, \mathfrak{k})$ is Hermitian follows from Lemma 3.1, so we need prove that it is irreducible. Note that $\mathfrak{p}^{\mathbb{C}}=\mathfrak{z} \oplus \overline{\mathfrak{z}}$. Moreover, Proposition 4.3 in [4] shows that $\operatorname{ad}(\mathfrak{h})$ preserves $\mathfrak{z}$ and the action of $\mathfrak{h}$ on $\mathfrak{z}$ is irreducible. This implies that ad(h) acts irreducibly on $\mathfrak{p}$. We now decompose $\mathfrak{h}=\mathfrak{k} \oplus \mathfrak{k}^{\prime}$ with respect to the Cartan Killing form $B$, where $\mathfrak{k}^{\prime}:=\mathfrak{k}^{\perp} \cap \mathfrak{h}$. We have

$$
B\left(\left[\mathfrak{k}^{\prime}, \mathfrak{p}\right], \mathfrak{p}\right)=B\left(\mathfrak{k}^{\prime},[\mathfrak{p}, \mathfrak{p}]\right)=0,
$$

and since $\left[\mathfrak{k}^{\prime}, \mathfrak{p}\right] \subseteq \mathfrak{p}$, we conclude that $\left[\mathfrak{k}^{\prime}, \mathfrak{p}\right]=\{0\}$. This means that the ad $(\mathfrak{k})$-action on $\mathfrak{p}$ is also irreducible and our first claim follows. Now $[\mathfrak{u}, \mathfrak{u}]=\mathfrak{u}$ so that $\mathfrak{u}$ is semisimple. Since $\mathfrak{k}$ acts on $\mathfrak{p}$ irreducibly and the symmetric pair is Hermitian, we immediately see that $\mathfrak{u}$ is simple (see e.g. Proposition 7.5 in [7]).

Remark 3.4. From the characterization of $\mathfrak{p}^{\mathbb{C}} \cap R_{\mathfrak{m}}^{+}$, we see that $\mathfrak{p}^{\mathbb{C}}$ contains the root space $\mathfrak{g}_{\theta}$, where $\theta \in R$ is the highest root. Actually, $\theta$ is the highest weight for the irreducible representation of $H$ on $\mathfrak{z}$. This gives a way for detecting the Lie algebra $\mathfrak{u}$ out of the painted Dynkin diagram $D$ of the flag manifold $G / H$ (see, e.g., [1], [3] for a detailed exposition). Indeed we can consider the painted Dynkin 
diagram corresponding to the given flag manifold $G / H$, in which the Dynkin diagram of the semisimple part of $\mathfrak{h}^{\mathbb{C}}$ is obtained by deleting some (black) nodes in the Dynkin diagram of $\mathfrak{g}^{\mathbb{C}}$. We can then embed $D$ into the extended Dynkin dia$\operatorname{gram} \tilde{D}$ of $\mathfrak{g}^{\mathbb{C}}$ and we see that the Dynkin diagram of $\mathfrak{u}^{\mathbb{C}}$ is given by the connected component containing $-\theta$ of the complement in $\tilde{D}$ of the black nodes (see also the discussion on p. 88 in [4]).

Now we can define the subalgebra

$$
\mathfrak{h}^{\prime}:=\mathfrak{h} \oplus \mathfrak{p}
$$

and note that the corresponding connected Lie subgroup $H^{\prime} \subset G$ is compact, because $\mathfrak{h}^{\prime}$ has maximal rank. Indeed the closure $\bar{H}^{\prime}$ is connected and has Lie algebra $\overline{\mathfrak{h}}^{\prime}$ which normalizes $\mathfrak{h}^{\prime}$. Therefore we have the $B$-orthogonal decomposition $\overline{\mathfrak{h}}^{\prime}=\mathfrak{h}^{\prime} \oplus \mathfrak{f}$ with $\left[\mathfrak{h}^{\prime}, \mathfrak{f}\right] \subseteq \mathfrak{h}^{\prime} \cap \mathfrak{f}=\{0\}$. It then follows that $\mathfrak{f}$ commutes with $\mathfrak{h}^{\prime}$, hence with the maximal abelian subalgebra contained in $\mathfrak{h} \subset \mathfrak{h}^{\prime}$. Therefore $\mathfrak{f} \subset \mathfrak{h}$, hence $\mathfrak{f}=\{0\}$ and $H^{\prime}=\bar{H}^{\prime}$.

The homogenous space $M^{\prime}=G / H^{\prime}$ is compact and has dimension given by the coindex of symmetry $k$. Note that in general the invariant complex structure on $M$ does not descend to an invariant (almost)-complex structure on $M^{\prime}$.

Lemma 3.5. We have $k \geq 6$.

Proof. Since $G$ is simple, its action on $M^{\prime}$ is almost effective and therefore $\operatorname{dim} G \leq$ $\frac{1}{2} k(k+1)$. Note that $k$ is an even integer. In [2] it is proved that $k=2$ implies that $\operatorname{dim} M=3$, so that in our case $k \geq 4$. We now show that $k=4$ can be ruled out. Indeed in this case we have $\operatorname{dim} G \leq 10$ and being $G$ simple, we have $\mathfrak{g}=\mathfrak{s u}(3)$ or $\mathfrak{s o}(5) \cong \mathfrak{s p}(2)$. In any case all the flag manifolds with isometry group $\mathrm{SU}(3)$ or $\mathrm{SO}(5)$ are Hermitian symmetric spaces (note that $\mathrm{SO}(5) / \mathrm{U}(2) \cong \mathrm{SO}(6) / \mathrm{U}(3)$ ).

Lemma 3.6. We have

$$
\operatorname{dim} G \leq \frac{1}{2} k(k-1) .
$$

Proof. We have the general estimate $\operatorname{dim} G \leq \frac{1}{2} k(k+1)$, since $G$ acts almost effectively on $M$. We first show that equality can never occur. Indeed, it is well known (see e.g. [6]) that equality occurs precisely when $\mathfrak{g} \cong \mathfrak{s o}(k+1)$ and $\mathfrak{h}^{\prime} \cong \mathfrak{s o}(k)$. Note that $k \geq 6$ by Lemma 3.5 and therefore $\mathfrak{h}^{\prime}$ is simple. We note that $\mathfrak{h}^{\prime}=\mathfrak{k}^{\prime} \oplus \mathfrak{u}$ using the same notations as in the proof of Lemma 3.3. Moreover, $\left[\mathfrak{k}^{\prime}, \mathfrak{p}\right]=0$ implies that $\left[\mathfrak{k}^{\prime},[\mathfrak{p}, \mathfrak{p}]\right]=\left[\mathfrak{k}^{\prime}, \mathfrak{k}\right]=0$ by Jacobi, hence $\left[\mathfrak{k}^{\prime}, \mathfrak{u}\right]=\{0\}$ and $\mathfrak{k}^{\prime}$ is an ideal of $\mathfrak{h}^{\prime}$. Since $\mathfrak{h}^{\prime}$ is simple we see that $\mathfrak{k}^{\prime}=\{0\}$ and $\mathfrak{h}^{\prime}=\mathfrak{u} \cong \mathfrak{s o}(k)$. Now using the fact that $(\mathfrak{u}, \mathfrak{k})$ is an Hermitian symmetric pair, we have that $\mathfrak{h}=\mathfrak{k}$ is either $\mathbb{R} \oplus \mathfrak{s o}(k-2)$ or $\mathfrak{u}\left(\frac{k}{2}\right)$. Now only $\mathfrak{h}=\mathfrak{u}\left(\frac{k}{2}\right) \mathrm{c}$ an be the isotropy of a flag manifold with $\mathfrak{g}=\mathfrak{s o}(k+1)$ (see e.g. [3]) and again the only invariant Kähler metric on such flag manifold is the symmetric one.

A classical result (see e.g. Theorem 3.2 in [6]) states that the dimension $d$ of the isometry group of a $k$-dimensional manifold $(k \neq 4)$ is less or equal to $\frac{1}{2} k(k-1)+1$ whenever it is not equal to $\frac{1}{2} k(k+1)$. Moreover, when $d=\frac{1}{2} k(k-1)+1$, a complete classification of the manifold is achieved (see Theorem 3.3 in [6]), showing 
that the isotropy representation has always a non trivial fixed vector. Since $\mathfrak{h}^{\prime}$ has maximal rank, the isotropy representation of $\mathfrak{h}^{\prime}$ has no trivial submodule and therefore $\operatorname{dim} G \leq \frac{1}{2} k(k-1)$.

Lemma 3.7. We have that $k=6$ if and only if $\mathfrak{g}=\mathfrak{s u}(4), \mathfrak{h}=2 \mathbb{R} \oplus \mathfrak{s u}(2)$. In this case the leaves of symmetry are biholomorphic to $\mathbb{C} P^{2}=\mathrm{SU}(3) / \mathrm{U}(2)$.

Proof. By Lemma 3.6 we get $\operatorname{dim} G \leq 15$. Since $G$ is simple and using the arguments in the proof of Lemma 3.5, we see that $\mathfrak{g}=\mathfrak{s u}(4)$ or $\mathfrak{g}_{2}$. When $\mathfrak{g}=\mathfrak{s u}(4)$ we see that the only non-symmetric flag is the one with $\mathfrak{h}=2 \mathbb{R} \oplus \mathfrak{s u}(2)$ and a simple computation shows that $\mathfrak{u} \cong \mathfrak{s u}(3)$.

As for $\mathfrak{g}_{2}$, we have precisely two distinct flag manifolds with $G=G_{2}$, namely $M=G_{2} / H$ where $\mathfrak{h} \cong \mathfrak{u}(2)$ with semisimple part containing a long or a short root space. If $R_{\mathfrak{h}}$ consists of a long root, then $M \cong Q_{5}=\mathrm{SO}(7) / \mathrm{SO}(2) \times \mathrm{SO}(5)$, the metric is symmetric and $G_{2}$ is a proper subgroup of the full isometry group (see Theorem 2.1). If $R_{\mathfrak{h}}$ is given by a short root, then $M$ is the twistor space of the Wolf space $G_{2} / \mathrm{SO}(4)$. In this case it is easy to see that the module $\mathfrak{p}$ is 2-dimensional (corresponding to the fibres of the twistor fibration), hence $k=8$.

\section{References}

[1] Alekseevsky, D. V.: Flag manifolds. In: 11th Yugoslav Geometrical Seminar (Divcibare, 1996). Zb. Rad. Mat. Inst. Beograd. (N.S.) 6 (14) (1997), 3-35.

[2] Berndt, J., Olmos, C. and Reggiani, S.: Compact homogeneous Riemannian manifolds with low co-index of symmetry. To appear in J. Eur. Math. Soc. (JEMS).

[3] Bordermann, M., Forger, M. And Römer, H.: Homogeneous Kähler manifolds: paving the way towards new supersymmetric sigma models. Comm. Math. Phys. 102 (1986), 605-647.

[4] Burstall, F. E. And Rawnsley, J. H.: Twistor theory for Riemannian symmetric spaces. Lecture Notes in Mathematics 1424, Spinger Verlag, 1990.

[5] Helgason, S.: Differential geometry, Lie groups, and symmetric spaces. Pure and Applied Mathematics 80, Academic Press, New York-London, 1978.

[6] Kobayashi, S.: Transformation groups in differential geometry. Classics in Mathematics 70, Springer Verlag, 1995.

[7] Kobayashi, S. and Nomizu, K.: Foundations of differential geometry Vol II. Interscience Publishers, John Wiley \& Sons, 1969.

[8] Olmos, C., Reggiani, S. and Tamaru, H.: The index of symmetry of compact naturally reductive spaces. Math. Z. 277 (2014), no. 3-4, 611-628.

[9] Onishchik, A. L.: Topology of transitive transformation groups. Johann Ambrosius Barth Verlag GmbH, Leipzig, 1994.

Received January 19, 2014.

Fabio Podestà: Dipartimento di Matematica e Informatica "U. Dini", Viale Morgagni 67/A, 50141 Firenze, Italy.

E-mail: podesta@unifi.it 